La universidad en el siglo XXI

Ailén Stranges

Actas de Periodismo y Comunicación, Vol. 3, N.o 1, diciembre 2017

ISSN 2469-0910 | http://perio.unlp.edu.ar/ojs/index.php/actas

FPyCS | Universidad Nacional de La Plata

La Plata | Buenos Aires | Argentina

\title{
La universidad en el siglo XXI
}

\author{
Ailén Stranges \\ strangesailen@gmail.com \\ Centro de Investigación de Lectura y Escritura (CILE) \\ Facultad de Periodismo y Comunicación Social \\ Universidad Nacional de La Plata \\ Argentina
}

La universidad, como institución del Estado, no puede nunca escindirse de su rol primero: aportar directamente al bienestar y al desarrollo de los integrantes de la sociedad. Cada una de sus unidades académicas debe pensar en la formación de profesionales que sepan desenvolverse en el marco de la crisis, que la entiendan y que diseñen soluciones consensuadas con los protagonistas que participan de ese contexto.

Asimismo, hay una realidad afuera de la cual hacerse cargo. Se debe pensar en la formación de profesionales preparados para el desarrollo de procesos de transformación. Por otra parte, supone la toma de un compromiso que debe ser asumido desde todos los actores y manifestarse en los procesos de construcción de contenidos y formación, articulados entre sí (Altamirano, 2007).

En este contexto y desde esas premisas, me propuse realizar, en el marco del Trabajo Integrador Final de grado de la Licenciatura en Comunicación Social, una herramienta capaz de aportar, integrar y fortalecer procesos de lectoescritura en el ingreso a la universidad.

El proyecto consistió en realizar una página web que plasmara el material teórico para todos aquellos estudiantes que deseen/necesiten acceder al Seminario-Taller de Prácticas del Lenguaje (reglas ortográficas, de acentuación, etc.); dentro de la cual se encuentra el curso práctico del Seminario-Taller en las Aulas Virtual UNLP al que tienen acceso (con usuario y contraseña) los alumnos que requieren trabajar de forma personalizada.

La propuesta de este Seminario-Taller trabajó sobre las herramientas normativas y gramaticales necesarias para la escritura que se desarrolla en el Taller de Comprensión y Producción de Textos I, material anual del primer año de la licenciatura en 
Comunicación Social. A partir de la página web, se puede acceder al curso de aulas web de la UNLP, donde se tratan de modo específico actividades, según la necesidad particular de cada alumno.

El impulso de las tecnologías de la información y comunicación en el mundo contemporáneo está produciendo un cambio cultural profundo cuyas consecuencias y alcances no son fáciles de vislumbrar. Se trata, eso sí, no sólo ya de un progreso instrumental, sino de un cambio de paradigma en la vida social, con especial impacto en la creación, circulación y aprovechamiento del conocimiento. Esto atañe e interpela profundamente a las universidades, que tienen en sus propias manos su desarrollo institucional. La virtualidad será, tarde o temprano, una condición que las atraviese, constituyendo un nuevo carácter, signado por la imbricación entre las formas de organización, intercambio y socialización presenciales y virtuales (Sileoni, 2010).

Por esta razón, no hay que dejar de lado la importancia de incorporar las plataformas virtuales en las unidades académicas.

Las plataformas educativas vigentes en la Universidad se irán usando cada vez más en las cátedras como mecanismos de información y comunicación entre docentes y alumnos, disposición de materiales de clase directa y mediante enlaces con fuentes electrónicas de información, ejercitación, desarrollo de proyectos, seguimiento de la tarea de alumnos, lecturas dirigidas, etc. (Frangi, 2010: 116).

El director de Educación a Distancia de la Universidad Nacional de La Plata, Alejandro González ${ }^{1}$, sostuvo que la UNLP en mayo del 2016 tenía 1.645 aulas virtuales activas y 42.591 participantes activos (37.162 estudiantes y 5.429 docentes). Esto demuestra el compromiso y la voluntad por parte de las distintas unidades académicas de involucrarse y generar nuevas propuestas de enseñanza. Que la universidad sea bimodal (presencial y virtual) es sinónimo de inclusión y de oportunidades para todos y todas.

\section{La palabra escrita}

Sin duda gran parte de la labor profesional de todo periodista y/o comunicador social consiste en trabajar con la palabra escrita; si de hecho revisamos cualquier actividad tanto como periodista, planificador o docente, inmediatamente advertimos la

\footnotetext{
${ }^{1}$ En el marco de "I Jornadas sobre las prácticas docentes en la Universidad Pública" organizado por la Dirección de Capacitación y Docencia de la Universidad Nacional de La Plata. Fecha de realización: 7 y 8 de abril de 2016.
} 
importancia que tiene el texto escrito en las distintas formas de intervención profesional (Valentino, 2007).

El problema del que partí para crear la página web y el aula virtual tiene que ver con que en el imaginario de la educación superior se presume que los alumnos ingresan a la universidad con determinadas habilidades lectoescritoras. Y más aún en el caso de la carrera de Comunicación Social porque se cree que el alumno que elige este tipo de orientación es porque le gusta/sabe escribir todo tipo de texto de manera casi natural. Pero el tema es mucho más complejo que una cuestión instrumental.

Creo que la alfabetización académica es el lugar desde donde hay que comenzar a pensar esta problemática, y uno de los primeros pasos a seguir es revisar las prácticas de lectura y escritura que se vienen desarrollando en el ámbito de la educación superior. $Y$, para ello, es importante concebir al aprendizaje como un proceso de reelaboración, y no como un simple "corte y pegue" de información que pueden encontrar los estudiantes en internet.

\footnotetext{
"Caracterizar el aprendizaje en entornos virtuales como un proceso de construcción supone, esencialmente, afirmar que lo que el alumno aprende en un entorno virtual no es simplemente una copia o una reproducción de lo que en ese entorno se le presenta como contenido a aprender, sino una reelaboración de ese contenido mediada por la estructura cognitiva del aprendiz" (Onrubia, 2005: 3).
}

Ahí reside la importancia de asistir a los talleres extracurriculares que se ofrecen en la Facultad: Prácticas del Lenguaje presencial y digital, Español para Extranjeros y Encuentros Pedagógicos.

No son talleres remediales sino que partimos de considerar a la palabra escrita como una herramienta insoslayable en la formación del comunicador social. Por lo tanto, la reflexión y la ejercitación en el uso del lenguaje debe ser a lo largo de toda la formación académica; se aprende a escribir escribiendo (Valentino, 2007: 27).

Sin ir más lejos, creo que este es el camino para que la universidad sea de todos y todas. Creo que cada uno de nosotros, los estudiantes de las distintas unidades académicas, tenemos que devolverle algo de todo lo que la institución nos dio. Por eso, como sostuve anteriormente, propuse esta herramienta de inclusión.

\section{Alcances y limitaciones del Taller}


El hecho de tener, o no, computadora e internet marcaron los alcances y limitaciones del Taller de Prácticas del Lenguaje en AulasWeb. Si los alumnos cuentan con los medios y las herramientas para desarrollar el Seminario-Taller en formato digital, será una herramienta de gran alcance para que puedan realizar la ejercitación correspondiente. De lo contrario, será una limitación para poder hacerlo.

\footnotetext{
"La incorporación de las TIC a la educación está, pues, lejos de presentar un panorama tan homogéneo como se supone en ocasiones y sus efectos benéficos sobre la educación y la enseñanza distan de estar tan generalizados como se da a entender algunas veces, entre otras razones porque en la mayoría de los escenarios de educación formal y escolar las posibilidades de acceso y uso de estas tecnologías son todavía limitadas o incluso inexistentes" (Coll, 2008: 115).
}

Y será también una limitación si los estudiantes demoran en anotarse, ya que se pierde tiempo que se podría estar utilizando en realizar los ejercicios. Mencioné esto ya que para la inscripción virtual los jóvenes tienen que pasarle al docente el número de dni y un correo electrónico; luego, se los inscribe desde la Facultad y recién ahí ellos están en condiciones de habilitar su perfil y hacer uso del aula.

Como toda plataforma virtual requiere de compromiso por parte del estudiante ya que todo tiene tiempos pautados que hay que cumplir porque, de lo contrario, el proceso de enseñanza/aprendizaje no se desarrolla como se pensó en un comienzo.

\section{¿Para qué?}

Nunca hay que dejar de lado los objetivos que se tienen en la incorporación de las plataformas virtuales. Ya sea como un complemento de una materia presencial o bien como una materia $100 \%$ a distancia.

\footnotetext{
"La valoración del estado actual de la incorporación de las TIC a la educación formal y escolar y de su impacto sobre la enseñanza y el aprendizaje, así como las previsiones de futuro a este respecto, varía en función de la potencialidad educativa que se atribuye a estas tecnologías y también de los objetivos que se persiguen con su incorporación" (Coll, 2008: 124).
} 
Es importante conocer a los sujetos y tener en cuenta sus realidades, brindándoles la posibilidad de adquirir el capital cultural para la inclusión, la retención y la permanencia en el sistema educativo y que el acceso sea igual para todos es la meta fundamental. Darles la oportunidad que muchas veces no existe, para la inclusión no sólo educativa, sino también social. Y que tengan las herramientas para mejorar su escritura a través de Prácticas del Lenguaje Digital, es una nueva opción de aprendizaje pensada para todos/as.

Creo que es un aporte fundamental a la comunicación y a la educación porque permite el desarrollo de una herramienta clave para la inclusión. Como mencioné párrafos anteriores, la palabra es la herramienta de nosotros, los comunicadores. Y justamente como profesionales de la palabra tenemos que pensar estrategias para que cada vez más y más estudiantes formen parte del sistema educativo argentino: que lleguen, se queden y se gradúen.

Y si consideramos el contexto por el que estamos atravesados, utilizar las nuevas tecnologías y las herramientas que se brindan no sólo desde la Universidad, sino en todos los niveles educativos, aportan al desarrollo y desenvolvimiento de los estudiantes en las diversas carreras.

\section{Bibliografía}

- Altamirano, L. y Wahnón, G. (2007) "Encuentro de saberes" en Revista Trampas No56. La Plata: EPC. [en línea] Recuperado en: http://goo.gl/Zbu3Gu Fecha de consulta: 1 de junio de 2017.

- Aulas Web UNLP. Disponible en: https://aulasweb.ead.unlp.edu.ar/aulasweb/

- Coll, C. (2008). Los desafíos de las TIC para el cambio educativo. España: Fundación Santillana.

- Frangi, J. (2010). "Una universidad para las próximas décadas" en Revista de la Universidad Nro35. La Plata: EDULP.

- Onrubia, J. (2005). Aprender y enseñar en entornos virtuales: actividad conjunta, ayuda pedagógica y construcción del conocimiento. RED. Revista de Educación a Distancia, número monográfico II. [en línea] Recuperado en:

http://www.um.es/ead/red/M2/ Fecha de consulta: 1 de junio de 2017.

- Sileoni, A. (2010). "Un presente universitario" en Revista de la Universidad Nro. 35. La Plata: EDULP.

- Valentino, A. (2007). "Prácticas de lectura y escritura en la formación de comunicadores" en Revista Trampas No56. La Plata: EPC. [en línea] Recuperado en: http://goo.gl/vC5h5F Fecha de consulta: 1 de junio de 2017. 